05,01

\title{
Спонтанный спин-переориентационный переход в сплавах (NdSmDy)(FeCo)B
}

\author{
() А.И. Дмитриев \\ Институт проблем химической фризики РАН, \\ Черноголовка, Россия \\ E-mail: aid@icp.acru
}

(Поступила в Редакцию 25 мая 2016 г.

В окончательной редакции 7 июня 2016 г.)

В сплаве $(\mathrm{NdSmDy})(\mathrm{FeCo}) \mathrm{B}$ при температуре $T=110 \mathrm{~K}$ обнаружен магнитный переход, сопровождающийся резким уменьшением намагниченности. Установлено, что в образце происходит спин-переориентационный переход, сопровождающийся изменением типа магнитной анизотропии. Высоким температурам $T>110 \mathrm{~K}$ соответствует анизотропия типа „легкая ось“. При низких температурах $T<110 \mathrm{~K}$ формируется магнитная структура типа „конус осей легкого намагничивания“.

\section{1. Введение}

Одной из ключевых характеристик постоянного магнита является максимальное энергетическое произведение (запасенная магнитная энергия). Эта величина определяется параметрами петли магнитного гистерезиса: намагниченностью насыщения, остаточной намагниченностью и коэрцитивной силой. Теоретическое предельно высокое значение коэрцитивной силы определяется значениями константы магнитной анизотропии $K$ и намагниченности насыщения $M_{s}$ согласно выражению $H_{a}=2 K / M_{s}$ (где $H_{a}$ - поле магнитной анизотропии). Фундаментальной задачей увеличения коэрцитивной силы является создание магнитных материалов с высокой магнитной анизотропией. В сильно анизотропных магнитных материалах независимо от их формы, размеров и механизмов формирования магнитного гистерезиса имеется принципиальная возможность получить величину коэрцитивной силы, близкую по величине к полю магнитной анизотропии при остаточной намагниченности, равной намагниченности насыщения [1].

В настоящее время среди различных типов коммерческих постоянных магнитов наибольшее значение максимального энергетического произведения имеют редкоземельные магниты типа $\mathrm{Nd}_{2} \mathrm{Fe}_{14} \mathrm{~B}$ (фаза 2-14-1). Сплавы этой серии обладают тем преимуществом, что открывают новые возможности тонкой подстройки магнитных характеристик (поля анизотропии и соответственно коэрцитивной силы) путем варьирования состава (замены редкоземельных и переходных элементов). Конкурирующие вклады в полную магнитную анизотропию сплава нескольких различных ионов могут приводить к спонтанным спин-переориентационным переходам [2]. Температурная манипуляция направлением вектора намагниченности открывает новые возможности для применения сплавов типа $\mathrm{Nd}_{2} \mathrm{Fe}_{14} \mathrm{~B}$ в устройствах нанологики и спинтроники в условиях сильной анизотропии, когда магнитные поля, требуемые для пере- ключения направления намагниченности (а значит, изменения битового состояния), становятся неприемлемо большими.

Спин-переориентационный переход в сложных многокомпонентных сплавах $(\mathrm{NdSmDy})(\mathrm{FeCo}) \mathrm{B}$ обсуждался ранее в работе [2]. Резкое уменьшение магнитного момента и максимум на динамической магнитной восприимчивости в окрестности температуры $T=110 \mathrm{~K}$, обнаруженные в [2], сами по себе не свидетельствуют о том, что наблюдаемый магнитный фазовый переход является именно спин-переориентационным. Ссылки на другие работы, приведенные в [2], в данном случае очевидно недостаточны. По мнению авторов [2], „исследование намагниченности сплавов в постоянном магнитном поле несет очень опосредованную, трудно интерпретируемую информацию о магнитной анизотропии и ее вариациях“. Как показано в настоящей работе, строгий анализ полевых и температурных зависимостей намагниченности, измеренных в постоянных магнитных полях, является достаточным для квалификации спин-переориентационного перехода.

Целью работы является установление механизма и закономерностей магнитного фазового перехода, наблюдаемого в многокомпонентных сплавах (NdSmDy)(FeCo)B.

\section{2. Методика}

Образцы $\left(\mathrm{Nd}_{0.62} \mathrm{Sm}_{0.05} \mathrm{Dy}_{0.33}\right)_{16.2}\left(\mathrm{Fe}_{0.77} \mathrm{Co}_{0.23}\right)_{78.1} \mathrm{~B}_{5.7}$ в виде магнитно-текстурированных пластин размером $4 \times 2 \times 0.5 \mathrm{~mm}$ были получены методом прессования и спекания частиц сплава размером 500-600 $\mu \mathrm{m}$ в магнитном поле, задающем сонаправленную взаимную ориентацию осей легкого намагничивания отдельных зерен тетрагональной фазы 2-14-1. Легкая ось фазы 2-14-1 совпадает с кристаллографическим направлением [001]. Подробно методика приготовления и аттестации образцов методом микрорентгеноспектрального анализа с помощью анализатора JCMA-733 приведе- 
на в работах $[3,4]$. С помощью СКВИД-магнитометра MPMS 5XL Quantum Design при различных температурах были измерены зависимости магнитного момента от напряженности магнитного поля до $50 \mathrm{kOe}$. Также была измерена температурная зависимость магнитного момента в диапазоне температур $T=2-350 \mathrm{~K}$ в магнитном поле 20 Ое. Перед измерением образец охлаждался в нулевом магнитном поле. Во всех экспериментах вектор напряженности магнитного поля был направлен поперек длинного ребра пластины, соответствующего оси легкого намагничивания при комнатной температуре (ось с на вставке к рис. 1). Ось легкого намагничивания образца совпадала с направлением текстуры зерен.

\section{3. Экспериментальные результаты и обсуждение}

На рис. 1 приведена температурная зависимость намагниченности $M(T)$ образца, охлажденного в нулевом магнитном поле, измеренная в магнитном поле напряженностью 20 Ое. В окрестности температуры $T=110 \mathrm{~K}$ в образце происходит магнитный фазовый переход, сопровождающийся резким уменьшением намагниченности (рис. 1). Резкое изменение магнитного состояния также отражается на зависимостях намагниченности образца от напряженности магнитного поля $M(H)$ (рис. 2). При высоких температурах $(T>110 \mathrm{~K})$ вид зависимостей $M(H)$ близок к линейному, что и следовало ожидать, когда вектор напряженности магнитного поля направлен перпендикулярно оси легкого намагничивания. При низких температурах $T<110 \mathrm{~K}$ на зависимостях $M(H)$ обнаруживаются перегиб и тенденция к насыщению. Аналогичное поведение зависимостей $M(T)$ и $M(H)$ наблюдалось ранее в окрестности спин-переориентационного

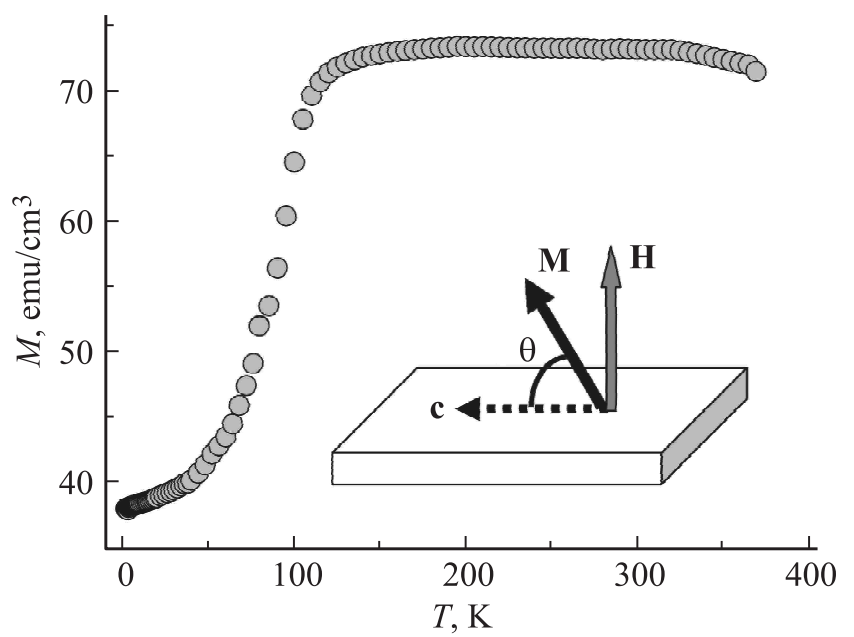

Pис. 1. Температурная зависимость намагниченности образца, охлажденного в нулевом магнитном поле, измеренная в магнитном поле напряженностью 20 Ое. На вставке - схема ориентации образца в экспериментах.

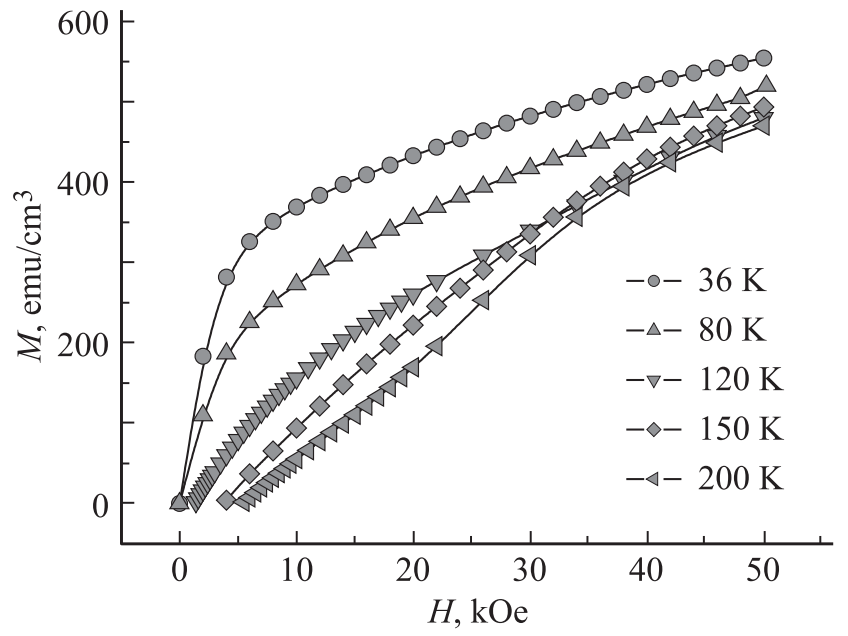

Рис. 2. Зависимости намагниченности образца от напряженности магнитного поля, измеренные при различных температурах.

перехода в нанопроволоках $\mathrm{In}_{0.24} \mathrm{Fe}_{1.76} \mathrm{O}_{3}$ [5]. Встречаются публикации, в которых обсуждается спинпереориентационный переход в редкоземельных магнитах типа $\mathrm{Nd}_{2} \mathrm{Fe}_{14} \mathrm{~B}[6,7]$.

Энергия $E$ магнитно-текстурированного образца, как и монокристалла, описывается выражением

$$
E=K_{1} \sin ^{2} \theta+K_{2} \sin ^{4} \theta-M_{s} H \cos (\varphi-\theta),
$$

где первые два слагаемых соответствуют энергии магнитной анизотропии $E_{a}$, а последнее слагаемое магнитостатической энергии. Здесь $K_{1}-$ константа магнитной анизотропии первого порядка, $K_{2}-$ константа магнитной анизотропии второго порядка, $M_{s}-$ намагниченность насыщения, $\theta-$ угол между осью с и вектором намагниченности (см. вставку на рис. 1), $\varphi$ - угол между осью с и вектором напряженности $\mathbf{H}$ магнитного поля (в наших экспериментах $\varphi=90^{\circ}$ ). Направление оси легкого намагничивания определяется соотношением констант магнитной анизотропии первого $K_{1}$ и второго $K_{2}$ порядков. Могут иметь место три случая [8]:

1) $K_{1}>0-$ анизотропия типа ,легкая ось“;

2) $K_{1}<0, K_{1}+2 K_{2}<0-$ анизотропия типа „легкая плоскость";

3) $K_{1}<0, K_{1}+2 K_{2}>0$ - анизотропия типа „конус легких осей“", направления осей легкого намагничивания образуют конус с углом раствора $2 \theta$ и высотой, ориентированной вдоль оси с.

Рассмотренные выше три случая прямо следуют из минимизации полной энергии образца $\partial E / \partial \theta=0$ :

$\frac{\partial E}{\partial \theta}=2 K_{1} \sin \theta \cos \theta+4 K_{2} \sin ^{3} \theta \cos \theta-M_{s} H \sin (\varphi-\theta)=0$.

Измеряемая с помощью СКВИД-магнитометра намагниченность образца есть проекция вектора намагниченно- 


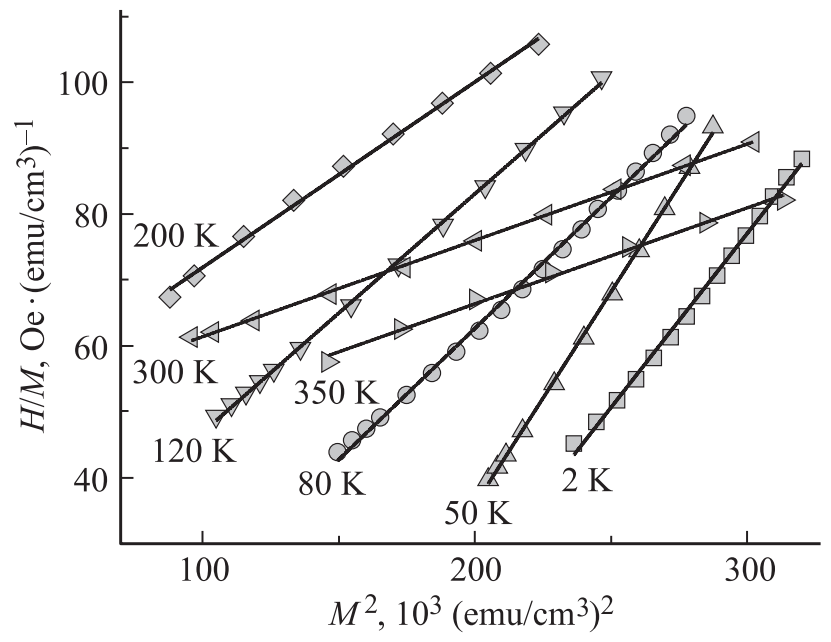

Рис. 3. Зависимости намагниченности образца от напряженности магнитного поля в координатах $H / M-M^{2}$. Сплошными линиями показаны аппроксимации.

сти насыщения на направление магнитного поля

$$
M=M_{s} \cos (\varphi-\theta)
$$

Подстановка (3) в выражение (2) с учетом того, что угол $\varphi=90^{\circ}$, приводит к уравнению

$$
\frac{4 K_{2}}{M_{s}^{4}} M^{2}+\frac{2 K_{1}}{M_{s}^{2}}=\frac{H}{M} .
$$

Из уравнения (4) следует, что зависимость намагниченности образца от напряженности магнитного поля $M(H)$, построенная в координатах $H / M-M^{2}$, должна быть прямой $y=k x+b$ с угловым коэффициентом $k=4 K_{2} / M_{s}^{4}$, $b=2 K_{1} / M_{s}^{2}$ (здесь $y=H / M, x=M^{2}$ ) [9].

На рис. 3 приведены зависимости намагниченности образца от напряженности магнитного поля в координатах $H / M-M^{2}$ при различных температурах. Сплошными линиями показаны аппроксимации уравнением прямой. Из аппроксимации получены коэффициенты $k$ и $b$, которые затем были пересчитаны в константы магнитной анизотропии первого $K_{1}$ и второго $K_{2}$ порядков для каждой температуры. Температурные зависимости констант магнитной анизотропии изображены на рис. 4. Видно, что в окрестности температуры $T_{\mathrm{SR}}=110 \mathrm{~K}$ константа $K_{1}$ меняет знак (рис. 4). Это означает, что при температуре $T_{\mathrm{SR}}=110 \mathrm{~K}$ в образце происходит спонтанный спин-переориентационный переход, связанный с изменением направления оси легкого намагничивания.

Сплошными линиями на рис. 4 показаны аппроксимации температурных зависимостей констант $K_{1}$ и $K_{2}$ модифицированной формулой Брюхатова-Киренского [10]

$$
K_{i}=K_{0 i} \exp \left(-a_{i} T^{2}\right)+b_{i}
$$

где значение $i=1$ соответствует константе анизотропии первого порядка $K_{1}$, а значение $i=2$ - константе анизотропии второго порядка $K_{2}$. Здесь $K_{0 i}, a, b-$ феноменологические константы (в формуле Брюхатова-Киренского, когда $b=0$, значение $K_{0 i}$ соответствует константе магнитной анизотропии при температуре $T=0 \mathrm{~K})$. Из аппроксимации были определены следующие значения этих констант: $K_{1}=-2.1 \cdot 10^{7} \mathrm{erg} / \mathrm{cm}^{3}$, $a_{1}=1.0 \cdot 10^{4} \mathrm{~K}^{-2}, \quad b_{1}=6.7 \cdot 10^{6} \mathrm{erg} / \mathrm{cm}^{3} ; \quad K_{2}=$ $=1.1 \cdot 10^{7} \mathrm{erg} / \mathrm{cm}^{3}, a_{2}=1.1 \cdot 10^{4} \mathrm{~K}^{-2}, b_{2}=3.1 \cdot 10^{6} \mathrm{erg} / \mathrm{cm}^{3}$. Столь высокие значения констант магнитной анизотропии указывают на то, что преобладающий вклад в магнитную анизотропию вносит кристаллографическая анизотропия. Согласно выражению $4 \pi M_{s}^{2}$ можно оценить вклад анизотропии формы, который не превышает $6 \cdot 10^{4} \mathrm{erg} / \mathrm{cm}^{3}$. На вставке к рис. 4 показаны схематические изображения эллипсоидов магнитной анизотропии, соответствующих анизотропии типа „легкая ось“ (справа) и „легкая плоскость“ (слева). Строго говоря, при температурах ниже температуры спин-переориентационного перехода $T_{\mathrm{SR}}$ направления осей легкого намагничивания образуют конус, так как выполняется условие $K_{1}+2 K_{2}>0$. Подтверждают это зависимости энергии магнитной анизотропии $E_{a}$ от угла между вектором намагниченности образца и осью с (рис. 5). При высоких температурах $(T>110 \mathrm{~K})$ зависимость $E_{a}$ имеет минимум при $\theta=0^{\circ}$ (и симметричный ему при $\theta=180^{\circ}$ ) (рис. 5), т.е. в отсутствие внешнего магнитного поля вектор намагниченности лежит вдоль оси с (анизотропии типа „легкая ось“). При низких температурах $(T<110 \mathrm{~K})$ минимумов на зависимости $E_{a}$ становится два: например, при температуре $T=60 \mathrm{~K}$ при углах $\theta=38$ и $142^{\circ}$ (и симметричные им при $\theta=218$ и $322^{\circ}$ ) (рис. 5). Это означает, что направления осей легкого

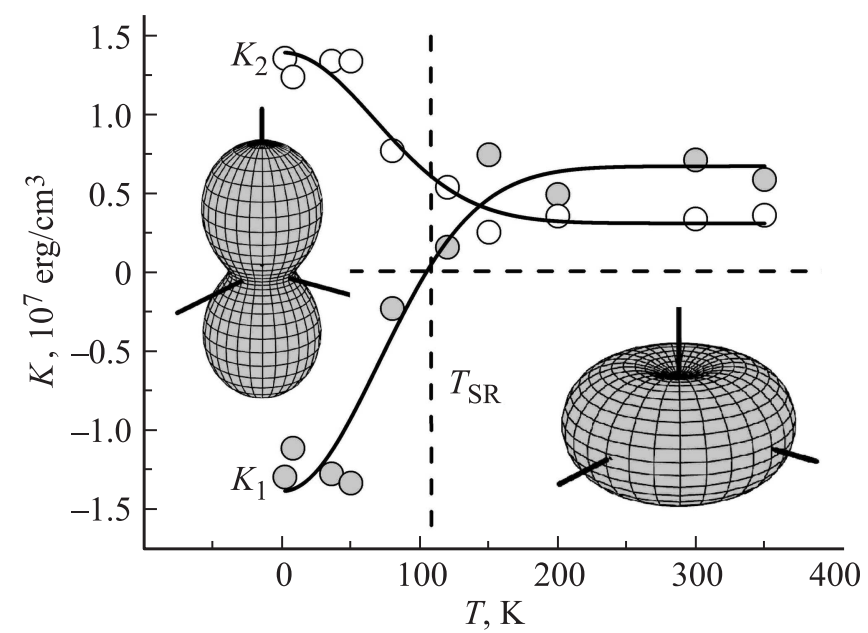

Рис. 4. Температурные зависимости констант магнитной анизотропии первого $K_{1}$ и второго $K_{2}$ порядков. Вертикальной штриховой линией отмечена температура спонтанного спинпереориентационного перехода $T_{\mathrm{SR}}$. На вставках показаны схематические изображения эллипсоидов магнитной анизотропии, соответствующих анизотропии типа „легкая ось“ (справа) и „легкая плоскость“ (слева). 


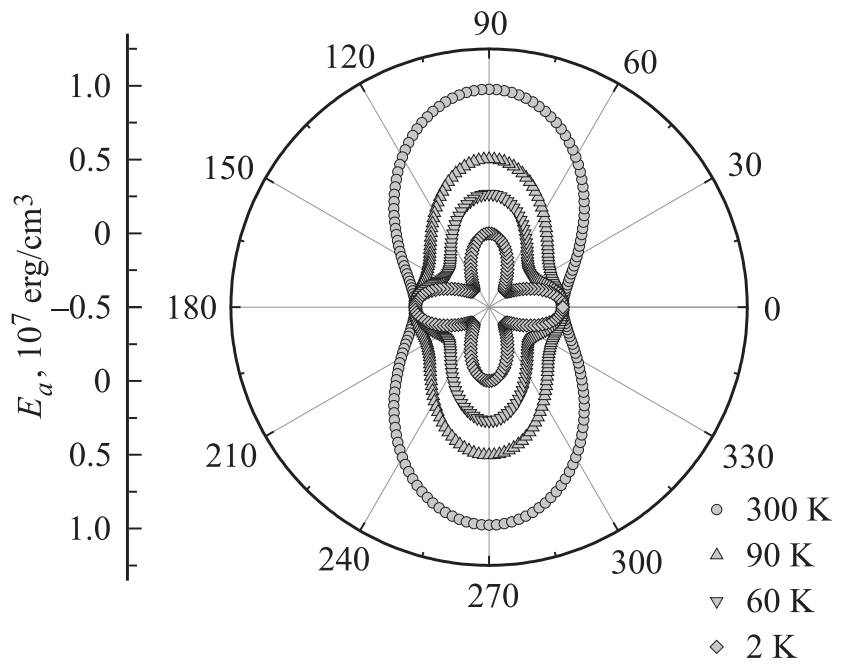

Рис. 5. Зависимости энергии магнитной анизотропии от угла между вектором намагниченности образца и осью с при различных температурах.

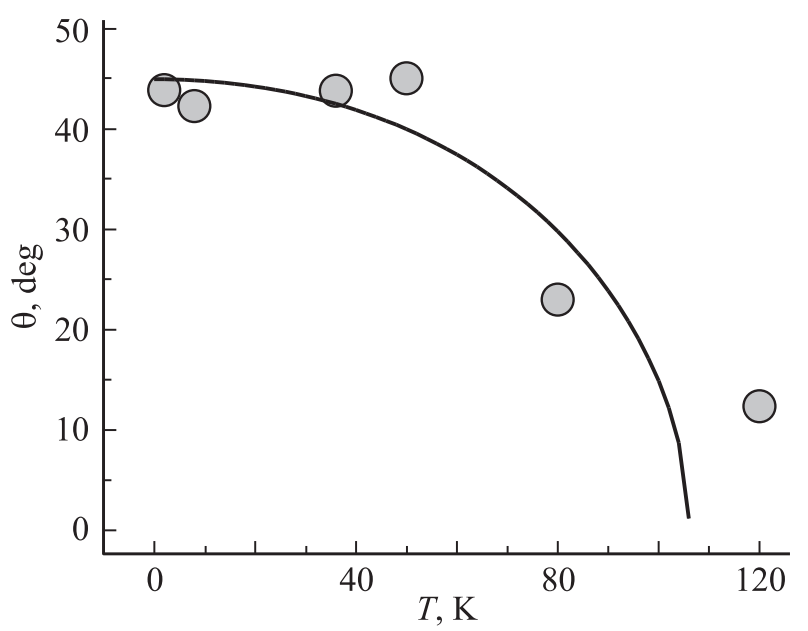

Рис. 6. Температурная зависимость угла между вектором намагниченности образца и осью с. Сплошной линией показана расчетная зависимость.

намагничивания образуют конус с углом раствора $76^{\circ}$ (анизотропии типа „конус легких осей“). Анизотропии типа „легкая плоскость“" в таком случае соответствовал бы конус с углом раствора $180^{\circ}$, т.е. когда $\theta=90^{\circ}$.

Угол $\theta$ определяется из минимизации энергии магнитной анизотропии образца $\partial E_{a} / \partial \theta=0[11]$

$$
\theta=\arcsin \sqrt{\frac{\left|K_{1}\right|}{2 K_{2}}}
$$

Зная температурные зависимости констант магнитной анизотропии первого $K_{1}$ и второго $K_{2}$ порядков, можно получить температурные зависимости угла $\theta$ (рис. 6). На рис. 6 сплошной линией показана расчетная кривая, полученная с использованием аппроксимирующих зависимостей (5). Таким образом, рис. 6 позволяет проследить за температурной эволюцией типа магнитной анизотропии в образце.

\section{4. Заключение}

Измерены зависимости намагниченности сплава $(\mathrm{NdSmDy})(\mathrm{FeCo}) \mathrm{B}$ от температуры $M(T)$ и напряженности магнитного поля $M(H)$. В окрестности температуры $T=110 \mathrm{~K}$ обнаружен магнитный фазовый переход, сопровождающийся резким уменьшением намагниченности образца. Анализ зависимостей $M(H)$, измеренных при различных температурах, позволил определить температурные зависимости констант анизотропии первого $K_{1}$ и второго $K_{2}$ порядков. Установлено, что при температуре $T=110 \mathrm{~K}$ константа $K_{1}$ изменят знак, что позволяет квалифицировать наблюдаемый магнитный переход как спин-переориентационный. Поворот оси легкого намагничивания сопровождается изменением типа магнитной анизотропии. Высоким температурам $T>110 \mathrm{~K}$ соответствует анизотропия типа „легкая ось“. При низких температурах $T<110 \mathrm{~K}$ формируется магнитная структура типа „конус осей легкого намагничивания“".

Автор признателен за предоставленные образцы Р.Б. Моргунову - учителю, многолетней работой с которым обусловлен интерес к теме, обсуждаемой в статье, и А.Д. Таланцеву за помощь в исследованиях на СКВИД-магнитометре.

\section{Список литературы}

[1] А.В. Дерягин. УФН 120, 393 (1976).

[2] Е.Н. Каблов, О.Г. Оспенникова, В.П. Пискорский, Д.В. Королев, Е.И. Куницына, А.Д. Таланцев, Р.Б. Моргунов. ФТТ 58, 1278 (2016).

[3] Е.Н. Каблов, В.П. Пискорский, Л.А. Брук. В сб.: Авиационные материалы. Изд-во МИСиС-ВИАМ, М. (2002). C. 191-197.

[4] В.П. Пискорский, Р.А. Валеев, Н.В. Назарова, Е.Б. Чабина, Е.А. Давыдова. В сб.: Горный информационноаналитический бюллетень. Изд-во МГГИ, М. (2007). С. $185-191$.

[5] А.И. Дмитриев, О.В. Коплак, А. Namai, Р.Б. Моргунов. ФTT 56, 1735 (2014).

[6] F.E. Pinkerton. J. Appl. Phys. 64, 5565 (1988).

[7] M. Foldeaki, L. Koszegi, R.A. Dunlap. J. Appl. Phys. 69, 5562 (1991).

[8] К.И. Белов, А.Е. Звездин, А.М. Кадомцева, Р.3. Левитин. УФН 119, 447 (1976).

[9] W. Sucksmith, J.E. Thompson. Proc. Roy. Soc. Lond. A 225, 362 (1954).

[10] Н.Л. Брюхатов, Л.В. Киренский. ЖЭТФ 6, 198 (1938).

[11] K.-D. Durst, H. Kronmuller. J. Magn. Magn. Mater. 59, 86 (1986). 